\title{
Abelian monopoles and center vortices in Yang-Mills plasma*
}

\author{
M. N. Chernodub \\ Laboratoire de Mathematiques et Physique Theorique CNRS UMR 6083, Fédération Denis \\ Poisson, Université de Tours, Parc de Grandmont, F37200, Tours, France \\ Institute of Theoretical and Experimental Physics ITEP, 117259 Moscow, Russia \\ E-mail: Maxim.Chernodub@lmpt.univ-tours.fr
}

\section{Atsushi Nakamura}

Research Institute for Information Science and Education, Hiroshima University,

Higashi-Hiroshima, 739-8527, Japan

E-mail: nakamura@rise.hiroshima-u.ac.jp

\section{I. Zakharov}

Institute of Theoretical and Experimental Physics ITEP, 117259 Moscow, Russia Max-Planck Institut für Physik, Föhringer Ring 6, 80805, München, Germany

E-mail: xxz@mppmu.mpg.de

Condensation of the Abelian monopoles and the center vortices leads to confinement of color in low temperature phase of Yang-Mills theory. We stress that these topological magnetic degrees of freedom are also very important in the deconfinement regime: at the point of the deconfinement phase transition both the monopoles and the vortices are released into the thermal vacuum contributing, in particular, to the equation of state and, definitely, to transport properties of the hot gluonic medium. Thus, we argue that a novel, magnetic component plays a crucial role. On the other hand, it was demonstrated that an effective three-dimensional description can be brought, beginning with high temperatures, down to the critical temperature by postulating existence of a system of $3 \mathrm{~d}$ Higgs fields. We propose to identify the $3 \mathrm{~d}$ color-singlet Higgs field with the $3 \mathrm{~d}$ projection of the $4 \mathrm{~d}$ magnetic vortices. Such identification fits well the $3 \mathrm{~d}$ properties of the theory and contributes to interpretation of the magnetic component of the Yang-Mills plasma.

8th Conference Quark Confinement and the Hadron Spectrum

September 1-6 2008

Mainz, Germany

${ }^{*}$ This work was supported by Grants-in-Aid for Scientific Research from "The Ministry of Education, Culture, Sports, Science and Technology of Japan" Nos. 17340080 and 20340055, JSPS Invitation Fellowship for Research in Japan, No. S-08035, by the STINT Institutional grant IG2004-2 025, and by the Federal Program of the Russian Ministry of Industry, Science and Technology No. 40.052.1.1.1112. The numerical simulations were performed using a SX-8 supercomputer at RCNP at Osaka University, and a SR11000 machine at Hiroshima University.

†peaker. 


\section{Introduction}

Both experimental observations at RHIC and numerical simulations of Yang-Mills theories indicate that the Yang-Mills plasma possesses quite unusual properties, for a review see, e.g., [1]. At temperatures just above the critical temperature $T_{c}$, the transport properties of the plasma correspond to an (ideal) fluid rather than to a weakly interacting gas. A reason for the liquid nature of the gluon plasma lies in a strong collective interaction of the gluons. A self-consistent theoretical explanation of this phenomena is still lacking and the topic attracts great interest nowadays.

Below we further discuss the picture $[2,3,4,5,6,7]$ according to which the unusual properties of the plasma appear as a result of a (topological) magnetic component of the gluon plasma. According to this picture monopolelike and vortexlike topological defects are responsible for the unusual thermodynamical and transport properties of the plasma. A brief review of the magnetic component of the plasma can be found in Ref. [3].

There are two constituents of magnetic component [2]: a particlelike magnetic monopole and a stringlike magnetic vortex. These constituents appear as singular magnetic defects in the gluon fields. The magnetic monopoles are related to the color confinement via the so called dual superconductor mechanism [8]. In the vortex picture the quark confinement emerges as a result of a percolation of the vortices [9].

The monopoles and the vortices are parts of a genuine non-Abelian object. For example, in $S U$ (2) gauge theory the center vortex can be regarded as an Abelian vortex carrying the magnetic flux which is equal to a half of the total magnetic flux of a monopole. The distribution of the magnetic part of the gluon energy density around a monopole is not spherical: each monopole is a source of two vortex fluxes which must be connected to other anti-monopole(s) because of a conservation of the vortex flux [10]. As a result, there appears a closed set of the vortex segments which connect alternating monopoles and antimonopoles, Figure 1. In $\mathrm{SU}\left(N_{c}\right)$ gauge theories the monopoles and vortices form nets [3]. Similar monopole-vortex chains were found in numerous (non-)supersymmetric non-Abelian gauge theories involving various Higgs fields [11].

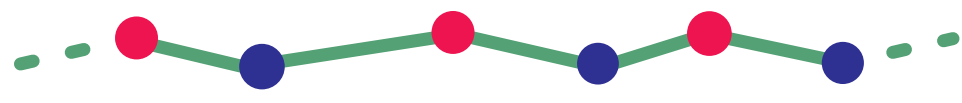

Figure 1: A part of a monopole-vortex chain in $S U(2)$ gauge theory.

A general phase diagram of the monopole component is suggested to be as follows [2]:

- $0<T<T_{c}$ : The monopoles form a condensate in the confinement phase.

- $T=T_{c}$ : The condensate melts into a monopole liquid at the phase transition.

- $T_{c}<T \lesssim 2 T_{c}$ : The liquid exists in the "strongly coupled" region in the deconfinement.

- $T \approx 2 T_{c}$ : The point of a gas-liquid crossover.

- $T \gtrsim 2 T_{c}$ : As temperature increases the monopole liquid gradually evaporates into a gas.

The intermediate monopole liquid state was also discussed in Refs. [2, 4] while the formation of the monopole gas at very high temperatures was predicted in Refs. [12, 13]. 


\section{Thermodynamics of Yang-Mills theories and topological objects}

This Section is based on results of Ref. [5]. Basic thermodynamical quantities of Yang-Mills theory can be found from the expectation value of the trace $\theta$ of the energy-momentum tensor $T_{\mu \nu}$ :

$$
\theta(T)=\left\langle T_{\mu}^{\mu}\right\rangle \equiv \varepsilon-3 p, \quad T_{\mu \nu}=2 \operatorname{Tr}\left[G_{\mu \sigma} G_{v \sigma}-\frac{1}{4} \delta_{\mu \nu} G_{\sigma \rho} G_{\sigma \rho}\right] .
$$

where $G_{\mu \nu}=G_{\mu \nu}^{a} t^{a}$ is the field strength tensor of the gluon fields $A_{\mu}$. For example, the pressure $p$, the energy density $\varepsilon$, and entropy $s$ can be calculated from the trace $\theta$ as follows:

$$
p(T)=T^{4} \int^{T} \frac{\mathrm{d} T_{1}}{T_{1}} \frac{\theta\left(T_{1}\right)}{T_{1}^{4}}, \quad \varepsilon(T)=3 p(T)+\theta(T), \quad s(T)=\frac{p(T)+\varepsilon(T)}{T} .
$$

The bare Yang-Mills theory is a conformal theory and therefore at the classical level the energy-momentum tensor is traceless. However, because of a dimensional transmutation the energy-momentum tensor exhibits a trace anomaly,

$$
\theta=\left\langle\tilde{\beta}(g) \operatorname{Tr} G_{\mu v}^{2}(x)\right\rangle, \quad \tilde{\beta}(g) \equiv \frac{\beta(g)}{g}=\frac{d \log g}{d \log \mu}=-g^{2}\left(b_{0}+b_{1} g^{2}+\ldots\right) .
$$

In Figure 2 (left) we show the trace anomaly (2.3) for SU(2) gauge theory. The measurements were performed for one lattice geometry, $18^{3} \times 4$, and the scaling properties of our results have not been studied yet. The trace of the energy-momentum tensor is subdivided into its electric (proportional to $\left.G_{4 i}^{2}, i=1,2,3\right)$ and magnetic $\left(G_{i j}^{2}, i, j=1,2,3\right)$ parts. In Figure 2 (right) we show the contribution
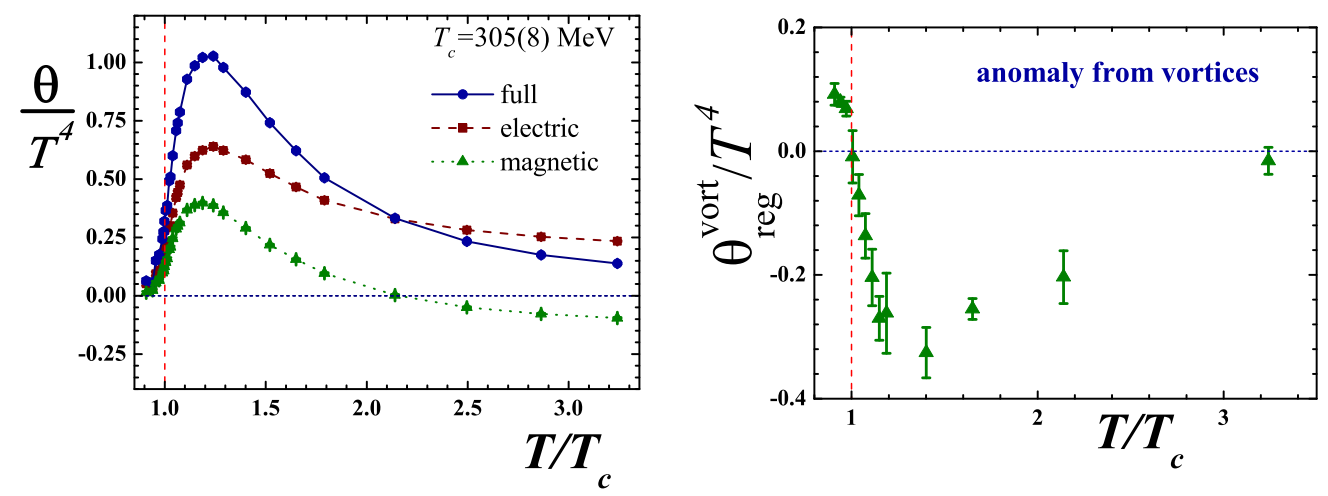

Figure 2: (left) The trace anomaly $\theta$ (in units of temperature) as a function of the temperature $T$ (in units of the critical temperature, $T_{c}$ ). The full anomaly (circles), and its electric (squares) and magnetic (triangles) contributions are shown. (right) The contribution of the magnetic vortices into the trace anomaly.

of the magnetic vortices into the trace anomaly of the gluon plasma. In the deconfinement phase just above the phase transition (i.e., in the monopole liquid region) the vortex-generated anomaly takes a negative value in agreement with general theoretical expectations [6].

Thus, the vortex constituents of the monopole-vortex chains are relevant for the thermodynamics. The monopole constituents are also important for thermodynamics [3, 5] as they carry an excess of the (magnetic part of) non-Abelian action density [14]. The action density, in turn, contributes to the trace anomaly (2.3) and, consequently, to the pressure and energy density of the Yang-Mills fields (2.2). 


\section{Effective 3d models of magnetic component}

In this Section we briefly compare the $4 \mathrm{~d}$, lattice-based picture of the magnetic component [2, 5] with newly developed $3 \mathrm{~d}$ models of the plasma. We find a close relation between the two approaches, originally developed in absolutely independent ways. The presentation is aimed to emphasize rather the general picture emerging rather than details.

It is quite common nowadays to assume that the dynamics of the Polyakov's lines plays $\Omega(\mathbf{x})$ a crucial role in the confinement-deconfinement phase transition $[15,16,17]$

$$
\Omega(\mathbf{x}) \equiv P \exp \left[-\int_{0}^{1 / T} d \tau A_{0}(\tau, \mathbf{x})\right],
$$

where $\tau$ is the Euclidean time. The vacuum expectation value, $\langle L\rangle$ with $L \equiv \frac{1}{2} \operatorname{Tr} \Omega$, serves as an order parameter, which is vanishing in the confinement phase and is finite in the deconfining phase. The symmetry - which is violated by a nonvanishing vacuum expectation value $\langle L\rangle$ - is the global $Z_{2}$ symmetry (for definiteness we consider $\mathrm{SU}(2)$ gauge group).

Note that $\Omega(\mathbf{x})$ depends only on $3 \mathrm{~d}$ variables and we are invited to consider $3 \mathrm{~d}$ reduced, or effective theories. A particular form of such a $Z_{2}$-symmetric lagrangian looks as [16] :

$$
L^{\mathrm{eff}}\left(A_{i}, \Omega\right)=\frac{1}{2} \operatorname{Tr} G_{i, j}^{2}+\frac{T^{2}}{g^{2}} \operatorname{Tr}\left|\Omega^{\dagger} D_{i} \Omega\right|^{2}+V\left(|\operatorname{Tr} \Omega|^{2}\right),
$$

where the potential $V\left(|\operatorname{Tr} \Omega|^{2}\right)$ can produce a non-trivial vacuum expectation value, $\langle L\rangle \neq 0$.

The Lagrangian (3.2) is nonrenormalizable in $3 \mathrm{~d}$. A renormalizable version of the effective Lagrangian was suggested in Ref. [17]. The idea can be represented as follows. There are two basic elements inherent to the construction (3.2). First, $Z_{2}$ invariance of the Lagrangian and, second, spontaneous breaking of the symmetry due to the potential. Both elements can be realized in terms of local fields, rather than non-local objects $\Omega$. To this end one introduces color triplet and color singlet $3 \mathrm{~d}$ scalar fields $\Pi_{a}$ and $\Sigma$. The Higgs-fields Lagrangian is then the standard kinetic terms plus the potential energy [17]:

$$
V\left(\Sigma, \Pi_{a}\right)=b_{1} \Sigma^{2}+b_{2} \Pi_{a}^{2}+c_{1} \Sigma^{4}+c_{2}\left(\Pi_{a}^{2}\right)^{2}+c_{3} \Sigma^{2} \Pi_{a}^{2},
$$

where the coefficients $b_{1,2}, c_{1,2,3}$ are organized in such a way that $\langle\Sigma\rangle \neq 0$. The success of the $3 \mathrm{~d}$ effective theory (3.3) is impressive both in terms of its numerical match to the original $4 \mathrm{~d}$ theory [17] and in the clarity of the underlying symmetry-based argumentation for its introduction.

We propose to identify the $3 \mathrm{~d}$ color-singlet with the $3 \mathrm{~d}$ projection of the magnetic vortices (or, of the $4 \mathrm{~d}$ magnetic component of the plasma) onto the $3 \mathrm{~d}$ space. We suggest that such an identification allows at least relate the newly made observations to the known properties of the $4 \mathrm{~d}$ magnetic component.

The vortices are $2 \mathrm{~d}$ surfaces percolating at low temperatures through the vacuum. The $3 \mathrm{~d}$ projection of the vortices is given by intersections of the $2 \mathrm{~d}$ surfaces with the $3 \mathrm{~d}$ time slice which are $1 \mathrm{~d}$ defects, or lines. These lines, or trajectories, are closed. The properties of these $1 \mathrm{~d}$ defects were studied in detail [18]. It was shown that properties of the $2 \mathrm{~d}$ and, consequently, of their $1 \mathrm{~d}$ projection depend crucially on the temperature in the vicinity of $T_{c}$. Namely, the surfaces become time-oriented at $T>T_{c}$ and do not percolate any longer from the $4 \mathrm{~d}$ point of view. However, 
as is emphasized in [18] the $3 \mathrm{~d}$ percolation continues, now in terms of the $1 \mathrm{~d}$ defects. Using the percolation theory one can readily argue, that the lattice observations imply the inequality $\left\langle\phi_{M}\right\rangle \neq 0$, where by $\phi_{M}$ we understand now a $3 \mathrm{~d}$ field corresponding to the cluster of the mentioned $1 \mathrm{~d}$ magnetic defects.

\section{References}

[1] M. Gyulassy and L. McLerran, Nucl. Phys. A 750 (2005) 30 [nucl-th/0405013]; E. V. Shuryak, Nucl. Phys. A 750 (2005) 64 [hep-ph/0405066].

[2] M. N. Chernodub and V. I. Zakharov, Phys. Rev. Lett. 98 (2007) 082002 [hep-ph/0611228] and in "Nagoya 2006, The origin of mass and strong coupling gauge theories", p. 80 [hep-ph/0702245].

[3] M. N. Chernodub and V. I. Zakharov, Phys.Atom.Nucl (2009), [arXiv:0806.2874 [hep-ph]].

[4] J. Liao and E. Shuryak, Phys. Rev. $\mathbf{C} 75$ (2007) 054907, [hep-ph/0611131]; Phys. Rev. Lett. 101 (2008) 162302, [arXiv:0804.0255 [hep-ph]].

[5] M.N. Chernodub, A. Nakamura and V.I. Zakharov, Phys. Rev. D78 (2008) 074021 [arXiv:0807.5012 [hep-lat]]; M.N. Chernodub, K. Ishiguro, A. Nakamura, T. Sekido, T. Suzuki, V.I. Zakharov, PoS LAT2007 174 (2007) [arXiv:0710.2547 [hep-lat]].

[6] A. Gorsky and V.I. Zakharov, Phys. Rev. D77 (2008) 045017, [arXiv:0707.1284 [hep-th]].

[7] A. D'Alessandro and M. D'Elia, Nucl. Phys. B 799 (2008) 241 [arXiv:0711.1266 [hep-lat]]; PoS(Confinement8)127 [arXiv:0812.1867 [hep-lat]].

[8] For reviews see, e.g., T. Suzuki, Nucl. Phys. Proc. Suppl. 30 (1993) 176; M. N. Chernodub and M. I. Polikarpov, in "Confinement, duality, and nonperturbative aspects of QCD", p. 387, ed. by P. van Baal, Plenum Press, 1997 [hep-th/9710205].

[9] For a review see, e.g., J. Greensite, Prog. Part. Nucl. Phys. 51 (2003) 1 [hep-lat/0301023].

[10] J. Ambjorn, J. Giedt and J. Greensite, JHEP 0002 (2000) 033 [hep-lat/9907021]; V. I. Zakharov, AIP Conf. Proc. 756 (2005) 182 [hep-ph/0501011].

[11] For reviews see, e.g., M. Shifman and A. Yung, Rev. Mod. Phys. 79 (2007) 1139 [hep-th/0703267]; D. Tong, arXiv:0809.5060 [hep-th].

[12] P. Giovannangeli, C. P. Korthals Altes, Nucl. Phys. B608 (2001) 203 [hep-ph/0102022]; C. P. Korthals Altes, in "Minneapolis 2006, Continuous advances in QCD", p. 266 [hep-ph/0607154].

[13] M. N. Chernodub, K. Ishiguro and T. Suzuki, JHEP 0309 (2003) 027 [hep-lat/0204003].

[14] B. L. G. Bakker, M. N. Chernodub and M. I. Polikarpov, Phys. Rev. Lett. 80 (1998) 30 [hep-lat/9706007]; M. N. Chernodub, A. D'Alessandro, M. D’Elia and V.I. Zakharov, in progress.

[15] A. M. Polyakov, Phys. Lett. B72 (1978) 477; A. Vuorinen, L.G. Yaffe, Phys. Rev. D74 (2006) 025011 [hep-ph/0604100].

[16] R. D. Pisarski, Prog. Theor. Phys. Suppl. 168 (2007) 276 [hep-ph/0612191].

[17] Ph. de Forcrand, A. Kurkela, A. Vuorinen, Phys.Rev. D77 (2008) 125014 [arXiv:0801.1566 [hep-ph]].

[18] M. N. Chernodub, M. I. Polikarpov, A. I. Veselov, M. A. Zubkov, Nucl. Phys. Proc. Suppl. 73 (1999) 575 [hep-lat/9809158]; R. Bertle, M. Faber, J. Greensite, S. Olejnik, JHEP 9903 (1999) 019 [hep-lat/9903023]; M. Engelhardt, K. Langfeld, H. Reinhardt, O. Tennert, Phys. Rev. D 61 (2000) 054504 [hep-lat/9904004]; J. Gattnar, K. Langfeld, A. Schafke, H. Reinhardt, Phys. Lett. B489 (2000) 251 [hep-lat/0005016]. 